\title{
EL MERCADO LABORAL Y LA RELIGIÓN: LA RELIGIÓN "VIVIDA" DE LA DIÁSPORA COMERCIAL DE OTAVALO (ECUADOR)
}

\section{LABOUR MARKET AND RELIGION: “LIVED" RELIGION IN THE CASE OF THE OTAVALO TRADE DIASPORA}

Dra. Rita SobczyK*, Dra. Rosa Soriano**, Dr. Andrés Caballero***

Resumen: La presente investigación muestra cómo el mercado laboral puede marcar profundamente las maneras en las que la religión es comprendida y practicada en el contexto migratorio. En la actualidad existen evidencias empíricas sólidas que indican que en diversos países de destino la población migrante, con frecuencia, se ve sobrerrepresentada en nichos laborales específicos. Este estudio, partiendo desde el análisis de caso de los kichwa otavalo, una población indígena del norte de Ecuador, revela cómo dicha concentración laboral puede plasmarse en el desarrollo de lo que denominamos la "religiosidad segmentada". Los resultados de la investigación cualitativa, realizada en el sur de España, evidencian que la comprensión de la vida religiosa en la emigración no puede llevarse a cabo sin el análisis longitudinal de los condicionantes socioeconómicos y políticos que repercuten en la inserción de los migrantes en los contextos de acogida.

* Profesora a Tiempo Completo, Dpto. de Ciencias Sociales, Universidad del Norte (Barranquilla, Colombia),rsobczyk@uninorte.edu.co

** Profesora Titular, Dpto. de Sociología, Universidad de Granada (España), rsoriano@ugr.es

*** Profesor a Tiempo Completo, Dpto. de Ciencias Sociales, Universidad del Norte (Barranquilla, Colombia), andrescaballero@uninorte.edu.co 
Palabras clave: Religión; mercado laboral; empresariado étnico; kichwa otavalo.

Abstract: This research demonstrates that the labour market mechanisms can profoundly influence the ways in which religion in understood and practiced in migratory contexts. Multiple studies, from diverse geographical contexts, show that migrants are often overrepresented in specific occupations. This paper, based on a case study of the kichwa otavalo, an indigenous population from Northern Ecuador, indicates that the concentration in one occupational niche can lead to the development of what we have called "segmented religiosity". The results of the qualitative research conducted in the South of Spain demonstrate that religious life in emigration cannot be properly understood without the longitudinal analysis of socioeconomic and political conditions that affect the insertion of migrants in the destination contexts. It is argued that, in spite of static conceptualization of religion, collective religious meanings and practices can change in a very short period of time in response to the requirements of the labour market.

Key words: Religion; labour market; ethnic entrepreneurship; kichwa otavalo.

\section{INTRODUCCIÓN}

La inserción laboral de la población migrante constituye una de las vertientes de mayor relevancia tanto en los estudios migratorios como en los debates públicos y políticos sobre la movilidad internacional. Pese a la gran heterogeneidad de las trayectorias profesionales de los migrantes y el aumento de los flujos de las personas altamente cualificadas (Bollard, McKenzie, Morten y Rapoport, 2011), múltiples investigaciones apuntan a que la población migrante, con frecuencia, está sobrerrepresentada en nichos laborales determinados (Colic-Peisker, 2010; Johnston, Khattab y Manley, 2015). En los países de Europa Occidental, tanto los migrantes como sus descendientes, tienden a caracterizarse por niveles más altos de desempleo y concentración en trabajos peor remunerados y de menor prestigio que la población receptora (Heath, Rothon y Kilpi, 2008; Koopmans, 2016). En el caso español se evidencia que, en los últimos veinticinco años, se ha generado una 
estructura ocupacional segmentada que afecta a la población nacida en el extranjero (Carrasco y García, 2015). En este contexto, una de las estrategias laborales desarrolladas por diversas comunidades migrantes es el empresariado étnico y el empresariado transnacional (Solé, Rubio y Cavalcanti, 2007; Portes, Guarnizo y Haller, 2002).

La presente investigación, basada en el estudio de caso de la diáspora comercial de Otavalo (Ecuador), muestra que los patrones de la inserción laboral de los migrantes deben ser tenidos en cuenta en el análisis del ámbito religioso. El estudio cualitativo realizado en el sur de España apunta al desarrollo de lo que denominamos la "religiosidad segmentada". El concepto denota la adherencia colectiva a significados y prácticas religiosas transformadas a raíz de la segregación en el mercado laboral. La religiosidad segmentada se da en estos casos en los que sobre-representación de migrantes de un origen en un nicho laboral determinado modifica la vida religiosa en función de los requisitos ocupacionales.

Los hallazgos de este estudio contribuyen a la comprensión de la diversidad religiosa en el contexto migratorio español. El auge migratorio de finales de los años 90 e inicios del siglo XXI ha contribuido ampliamente a la pluralización del panorama religioso existente. El estudio de esta realidad resulta necesario y urgente si tenemos en cuenta que, en múltiples países europeos, la religión desempeña un papel crucial en la delimitación de las fronteras sociales entre los migrantes y las sociedades de acogida (Foner y Alba, 2008; Zolberg y Woon, 1999). La percepción de la religión en términos esencialistas y estáticos repercute en la visión de las diferencias religiosas como obstáculos que dificultan la convivencia. El presente estudio demuestra, sin embargo, que la comprensión adecuada de los significados y prácticas religiosas debe partir de una perspectiva procesual y longitudinal, ya que solo de esta manera es posible captar el carácter dinámico del hecho religioso.

El presente artículo se inicia con una caracterización breve de la diáspora comercial de Otavalo, aportando una contextualización necesaria para la interpretación de los resultados. Seguidamente se desarrolla el sustento teórico del estudio, centrando en la perspectiva de la religión "vivida" y su utilidad para el análisis de los significados y prácticas religiosas en la emigración. Finalmente, se presentan los resultados del estudio, que demuestran el potencial del concepto de la "religiosidad segmentada" para el análisis de otras poblaciones migrantes. 


\section{LA DIÁSPORA COMERCIAL DE OTAVALO}

Los kichwa otavalo ${ }^{1}$ son uno de los grupos indígenas del norte de Ecuador, originarios de la ciudad de Otavalo y sus proximidades. Se trata de una población que se ha distinguido, desde tiempos preincaicos, por su tradición de comercio y producción de tejidos. Desde los años 70 ambas actividades se consolidaron como base de la economía étnica desarrollada en la región (Colloredo-Mansfeld, 1998). La venta de los productos artesanales y la creación artística en el ámbito de la música andina contribuyen a la transformación de Otavalo en un relevante centro turístico. Estas mismas actividades han sido emprendidas por muchos kichwa otavalo, que han emigrado de su zona de origen, sobre todo desde los años 90 (Meisch, 2002).

Las actividades emprendidas se ajustaron, en gran medida, al imaginario predominante sobre las poblaciones amerindias. Vendiendo las artesanías del origen y tocando música andina los empresarios kichwa otavalo respondieron a la demanda global de productos y servicios "étnicos". La búsqueda de la llamada "autenticidad cultural" y del encuentro con el "exótico Otro" se consolidaron como fundamentos de su éxito comercial.

Sin embargo, la prosperidad conseguida por los empresarios kichwa otavalo no sería posible sin la retención del control sobre el comercio de las artesanías producidas en Ecuador (Kyle, 2000). La eficacia de la estrategia económica empleada se basó en el abastecimiento directo en el país de origen y en el autoempleo, ambos sostenidos por fuertes lazos transnacionales. El interés científico en la diáspora comercial de Otavalo se ha plasmado en la recurrente citación de su caso como un ejemplo de empresariado transnacional (Portes et al., 2002; Zhou, 2004).

El éxito comercial de los emprendedores, tanto en Ecuador como en el exterior, repercutió ampliamente en el imaginario existente sobre los otavalos (Ordóñez, 2008). La ciudad de Otavalo pasó de ser un bastión del poder de la población no-indígena a un territorio "reconquistado" por los kichwa otavalo. Su éxito en

1 En la literatura existente no hay coherencia en los términos usados para referirse a la población estudiada. Sirvan de ilustración los siguientes ejemplos: los "kichwa Otavalo" (Torres, 2004), los "kichwa-otavalo" (Célleri et al., 2012), los "quichua-otavalo" (Conejo, Yamberla y Cachiguango, 2003).

En este texto utilizaremos los términos "kichwa otavalo" o, simplemente, "otavalos". 
el campo económico se vio acompañado del surgimiento de élites intelectuales, que jugaron un rol importante en el desarrollo del movimiento indígena en el país de origen.

Pese a la frecuente asociación de los kichwa otavalo a la prosperidad económica y el activismo político, no hay que olvidarse de las desigualdades internas que fracturan a esta población indígena. Tan solo una minoría de los kichwa otavalo pudo aprovechar la beneficiosa coyuntura económica (Kyle, 2000; Torres, 2004; Célleri y Jüssen, 2012).

Habría que enfatizar, además, que la concentración en los aspectos económicos y políticos no debe marginar la importancia de las potentes transformaciones socio-culturales e identitarias en el origen. El auge evangélico a nivel mundial afectó también, en gran medida, a las poblaciones indígenas del continente americano (Gumucio, 2002). En el caso de los otavalos, tuvo un importante impacto en el desarrollo de la especialización empresarial, su activismo político y la articulación de las relaciones intra y extragrupales (Korovkin, 1998; Lalander, 2010).

\section{LA PERSPECTIVA DE LA RELIGIÓN “VIVIDA" Y SUS IMPLICA- CIONES PARA LA METODOLOGÍA DE INVESTIGACIÓN}

La perspectiva de la religión "vivida" analiza la religión tal como esta es entendida y experimentada por los individuos en el ámbito cotidiano. Desde la publicación del volumen editado por David Hall (1997) "Lived religion in America: Towards a history of practice", el enfoque ha adquirido creciente relevancia en el análisis científico de la religión (McGuire, 2008; Schielke y Debevec, 2012; Woodhead, Dessing, Nielsen y Jeldtoft, 2014; Ammerman, 2016).

La consolidación de la perspectiva tiene que ver con lo que Schielke y Debevec (2012, p. 1) describen como "la compleja dualidad de la religión como práctica cotidiana y doctrina normativa" ${ }^{2}$. La vertiente normativa coexiste con las múltiples maneras en las que las personas definen y practican sus religiones en la vida diaria. La perspectiva de la religión "vivida" concibe a los actores sociales como sujetos

2 Traducción propia del texto original: "complex duality of religion as an everyday practice and a normative doctrine” (Schielke y Debevec, 2012, p. 1). 
activos que experimentan su religión en diversas y complejas formas, que pueden divergir de los discursos institucionales sobre la religión (McGuire, 2008). Sin embargo, estos últimos, frecuentemente, constituyen el único punto de partida para el estudio de la religión. Esto puede ser evidenciado, por ejemplo, en el uso de indicadores como la asistencia a la iglesia (Woodhead, 2014) o el seguimiento de rituales prescritos por las instituciones religiosas. Limitando el análisis de la vida religiosa al cumplimiento o no de la vertiente normativa establecida por las organizaciones, perdemos de vista todo el espectro de las experiencias religiosas que tienen lugar fuera de este marco. El enfoque de la religión "vivida" abarca la diversidad de actores sociales que participan en la construcción de los significados religiosos, minimizando la influencia del etnocentrismo y del esencialismo institucional en el análisis de la religión. Permite aproximarnos a los fenómenos religiosos con una gran sensibilidad a su diversidad y sus transformaciones en el tiempo.

La aplicación de esta perspectiva tiene implicaciones fundamentales en el ámbito metodológico. El enfoque que ubica el análisis de la religión en su contexto cotidiano permite comprender la influencia de heterogéneas expresiones religiosas en diversos contextos, como hospitales, entornos laborales, espacios de ocio, aulas escolares, etc. En la presente investigación, fruto de un prolongado estudio etnográfico realizado en varios periodos entre 2010 y 2014 en Villanueva ${ }^{3}$, una ciudad del Sur de España, el análisis desde la perspectiva de la religión vivida permitió captar la relación entre la religión y el ámbito laboral. A pesar de que el primer contacto con la población objeto de estudio tuvo lugar en una congregación evangélica a la que acude la mayoría de los otavalos asentados en la localidad, posteriormente la investigación fue ampliada para abarcar otros contextos en los que se desarrolla su vida cotidiana en la emigración. Aunque existe una minoría católica, la mayoría de los otavalos en Villanueva son evangélicos y se insertan dentro de una iglesia independiente e interdenominacional.

Las técnicas de recogida de datos fueron, sobre todo, la entrevista en profundidad y la observación participante. La comunicación con los informantes se llevó a cabo en castellano. Persiguiendo un mayor grado de apertura a los posibles significados y prácticas religiosas se

Con el objetivo de proteger el anonimato de los informantes, se ha cambiado el nombre real de la localidad de estudio. 
empleó un guión de entrevistas flexible. Su nivel de estructuración aumentó en las etapas de investigación más avanzadas. La observación participante facilitó la inmersión en la vida cotidiana de los informantes y la captación de la vinculación entre la religión y otros ámbitos de la experiencia migratoria.

Se empleó el muestreo teórico, seleccionando los perfiles de los informantes conforme iba desarrollándose la investigación. Se persiguió reflejar en la muestra final la diversidad de perfiles socio-demográficos de la población kichwa otavalo asentada en la localidad del estudio. La comunidad de Villanueva está formada principalmente por parejas jóvenes con hijos. Contrastando con la prevalencia de los varones entre los otavalos captada por investigaciones realizadas en otros contextos (Kyle, 2000), en Villanueva la estructura de sexos es equilibrada. El establecimiento de vínculos familiares en el destino y el hecho que la mayoría de parejas tuviera hijos en la emigración pueden ser muestra de la tendencia hacia el asentamiento en España. La mayoría de los informantes llegó a Europa entre la segunda mitad de los años 90 y principios del siglo XXI. Este tiempo, relativamente prolongado, transcurrido desde la inmigración permitió a la mayoría regularizar su situación administrativa y obtener cierta estabilidad económica.

\section{LA MIGRACIÓN Y EL ESPÍRITU EMPRESARIAL}

Para poder comprender la vida religiosa de la población analizada es imprescindible conocer su inserción laboral en España. La manera en la que los kichwa otavalo participan en el mercado de empleo en el destino se vincula estrechamente con el contexto del país de origen. El desarrollo de la economía étnica en Otavalo impulsó unas migraciones que continuaron con la estrategia empresarial se había mostrado exitosa en Ecuador. Los pioneros de los flujos transatlánticos eran empresarios kichwa otavalo que se involucraron en la venta de las artesanías del origen y en la creación en el campo de la música andina. El trabajo como empresarios autónomos, pese a tener sus riesgos, constituye una manera de obtener ingresos sin depender de los empleadores. La larga historia de explotación de mano de obra indígena y de discriminación en el origen repercute en que el trabajo como autónomo sea percibido como una estrategia que minimiza la discriminación en el mercado de empleo. 
"El indígena (...) hace mucho tiempo atrás ha sido (...) pisoteado por la gente blanca, por antiguos terratenientes (...) que siempre te obligaban a trabajar por tener una ración de comida (...) Nosotros que habiamos visto que (...) no está bien trabajar para alguien, sino si tienes la posibilidad de ser un trabajador por tu cuenta... ha sido mejor (...) Por eso la gente, aunque no gane mucho, siempre ha querido ser dueño de su trabajo (...) Los indígenas en nuestro país han sido casi esclavizados (...) Hubo una revolución muy grande para poder ser libres y poder ser cada uno dueño de sí mismo y desde este entonces el indígena comienza a tener su trabajo a su manera, deja de ser trabajador de otras personas" (Óscar)

El capital social constituye un elemento clave que permite la inserción en el nicho laboral ocupado por los migrantes kichwa otavalo (Kyle, 2001; Célleri et al., 2012). Los lazos transnacionales entre el origen y los múltiples lugares de destino de la migración de los emprendedores pioneros facilitaron la llegada de los sucesivos migrantes de Otavalo, articulándose un eficiente sistema de migración en cadena. A raíz de la adopción de una estrategia comercial compartida, surgió un alto nivel de homogeneidad laboral. Múltiples estudios analizan el exitoso modelo basado en la dedicación al comercio de las artesanías de Otavalo y la música folclórica (Kyle, 2000; Meisch, 2002).

Sin embargo, el presente estudio muestra que, en el sur de España, las oleadas migratorias alimentadas de las visiones románticas de la población indígena parecen ya formar parte del pasado. Esto no implica la erosión del espíritu emprendedor; todo lo contrario. Cuando la coyuntura del mercado cambia, los músicos y comerciantes kichwa otavalo se ajustan a la nueva realidad. Por esta razón podemos diferenciar dos fases principales en sus flujos migratorios. La primera se caracterizó por prácticas transnacionales intensas mantenidas gracias al comercio de las artesanías traídas desde el origen. Hay que enfatizar que, ya en esta primera fase, en el contexto de destino, se plasmaron las desigualdades socioeconómicas arrastradas desde el origen, traduciéndose en la diferenciación entre los empresarios kichwa otavalo y sus empleados contratados en el origen (Atienza, 2009) ${ }^{4}$. Estos primeros procedían de las élites de

4 Según David Atienza (2009), la celebración de Inti Raymi en la diáspora constituye una de las formas cruciales de lidiar con las tensiones interétnicas originadas a raíz de las desigualdades internas. 
tejedores y comerciantes otavalos que, a través de la migración, expandían sus negocios. Sus empleados migraban en gran medida debido a la escasez de las oportunidades en Ecuador. A pesar de contar con pocos recursos, podían migrar bien como empleados de los comerciantes o bien como músicos folclóricos. Esta segunda actividad no requería de una inversión inicial elevada. Se trataba de una época dominada por las migraciones de los varones, cuya inserción laboral en el destino se vinculaba con una alta movilidad en busca de mercados para las artesanías y público para la creación artística.

En este punto, siguiendo a Luz Caicedo (2010), hay que advertir que, pese a que podemos diferenciar dos grupos de migrantes, los empresarios y sus empleados, la realidad de estos primeros difícilmente puede ser descrita en términos de prosperidad. Aunque esta población, en general, disfrutaba de una situación mejor que la de sus empleados, la continuidad de sus negocios requería un trabajo duro que no necesariamente aseguraba la estabilidad económica.

El presente estudio muestra el surgimiento de una segunda fase de migraciones de los kichwa otavalo. En contraste con el predominio de la movilidad de los varones entre los pioneros de la movilidad transatlántica, hoy en día el balance entre sexos en la localidad de estudio es equilibrado. Pero el cambio que quizás más llama la atención tiene que ver con la desvinculación de la venta de artesanías y la música andina. Pese a que el famoso empresariado transnacional entró en decadencia, la gran mayoría de esta comunidad sigue dedicándose al comercio. Emprenden viajes comerciales, principalmente en el periodo de ferias, recorriendo los pueblos y ciudades de, sobre todo, el sur de España. Una minoría realiza viajes comerciales más lejanos, dirigiéndose también a otros estados europeos. El trabajo en otros ámbitos constituye algo muy excepcional, si bien algunos informantes indicaron que, en otras partes de España, hay más otavalos empleados como trabajadores asalariados.

La predominante dedicación al comercio pudo mantenerse gracias al empleo de estrategias que permitieron adaptarse a los cambios del mercado. Los productos ofrecidos en la actualidad se alejan profundamente de los que originaron las migraciones de los otavalos en el pasado. Hoy se pretende seguir las últimas modas de la sociedad de destino, vendiendo productos como bolsos, bisutería 
o ropa. La capitalización de la imagen de los indígenas amerindios, tan característica para la primera fase de las migraciones de los otavalos, contrasta con la realidad actual, plasmada en testimonios como el siguiente:

"Cada año va cambiando un poquito (...) Antes vendiamos artesanía y esto se vendía bien (...) Lo traían o lo comprábamos en Madrid (...) Pero ya no sale mucho (...) y por eso no trabajan con eso ya" (Enrique)

En esta reorientación de la estrategia empresarial tuvo gran relevancia el impacto de la crisis económica que afecta a España durante la última década. El reciente texto de Cebrián, Jiménez y Resino (2016) documenta la modificación de las estrategias comerciales en diversos tipos de negocios extranjeros. En el caso de los otavalos, el descenso del poder adquisitivo de la población española, unido a la menor rentabilidad de la importación de los productos desde Ecuador, empujó a los otavalos a buscar otras maneras de mantener sus proyectos empresariales.

La desvinculación de las actividades comerciales basadas en los lazos transnacionales mantenidos con el origen no suele ser percibida con nostalgia. Al poder mantener su trabajo como comerciantes y su autodefinición en estos términos, se enfatiza que las cualidades necesarias de los empresarios requieren desarrollar posturas innovadoras y creativas. La adaptación a nuevas condiciones del mercado es percibida predominantemente como un paso totalmente coherente con la estrategia laboral adoptada desde el inicio de su movilidad internacional. El orgullo del éxito de las ventas de las artesanías del origen, aunque presente, se ve eclipsado por las preocupaciones económicas de la vida diaria. Conviene recordar que el objetivo principal de las migraciones anteriores no era promocionar el patrimonio cultural del origen sino, sobre todo, sostenerse económicamente. Es cierto que múltiples estudios muestran que el desarrollo de la economía étnica en el origen contribuyó a una mayor concienciación grupal y, entre otros, al reclamo de los derechos de la población indígena en Ecuador (Huarcaya, 2011). Sin embargo, en el caso de la población kichwa otavalo asentada tanto en la zona de estudio como en otras partes de España (Ruiz, 2015), destaca la importancia fundamental atribuida a su autoidentificación como comerciantes, más allá de los productos que ofrezcan. Aunque anteriormente podían beneficiarse 
del mercado para productos "exóticos", hoy en día sus productos ya no son tan distintivos. No obstante, su concentración en el nicho laboral del comercio ambulante permanece intacta.

\section{TRAYECTORIAS LABORALES Y LA RELIGIÓN “VIVIDA”}

Para comprender las pautas de la religiosidad segmentada tenemos que adentrarnos en las trayectorias laborales de los kichwa otavalo de Villanueva. Se trata de una población caracterizada en la actualidad por la mayoritaria consolidación de los proyectos migratorios. Conviene recordar que el grueso de los otavalos de la localidad migró a Europa entre la segunda mitad de los años 90 y los inicios del siglo XXI. Sobre todo en el caso de las personas que vinieron con contratos de trabajo, se puede apreciar que el primer periodo, caracterizado por precariedad económica y la alta movilidad, ha dado paso a una mayor estabilidad y al asentamiento. A pesar de los viajes realizados en la temporada de trabajo, que tiene lugar en el periodo de las ferias, la mayoría de las personas regresa a su base en Villanueva en invierno. La posibilidad de empezar sus propios negocios, pasando de empleados a autónomos, fue crucial en este proceso de estabilización. Sirva como ejemplo el caso de Fabián que, tras múltiples viajes, consigue establecerse en Villanueva:

"Yo primera vez cuando llegué... había un paisano mío, así mismo me trajo [con] contrato para hacer música. Primera vez me fui a Bélgica y (...) estábamos por allí, unos 2 meses (...) y luego el jefe (...) no me daba la comida, solo hacia trabajar, trabajar (...) Por eso (...) cuando mis hermanos estaban en Suiza, lo comentamos con mis hermanos (...) y ellos vinieron a Bélgica a llevarme con su coche y alli yo me separé (...) del grupo. Me fui con mi hermano y estaba en Suiza trabajando unos 2 meses (...) Después unos primos (...) nos fuimos a (...) República Checa (...) Después de estar allí un rato, pasamos a Eslovaquia. Estábamos en Eslovaquia poco, haciendo música (...) después volvimos a Austria (...) De Austria bajamos a Suiza otra vez (...) De Suiza, como vivía mi hermana aquí en Villanueva, (...) dijo que venga acá, por eso nosotros bajamos de Suiza (...) En Suiza como no teníamos (...) piso, dormíamos sólo en el coche (...) estaba muy duro para mí (...) Acá venimos (...) mi hermana tenía un piso, dormíamos en el piso. 
Aquí más tranquilo que Suiza. Allá mucho control. No podíamos vivir tranquilos. Siempre teniendo miedo a la policía (...) no podíamos vivir tranquilos, como que si fuéramos un ladrón. Teníamos miedo (...) No teníamos nada de papeles también (...) Bajamos acá y solicitamos la residencia" (Fabián)

Las difíciles condiciones de trabajo y los problemas legales, que generaban una fuerte incertidumbre sobre el futuro, así como las fracturas internas basadas en la diferenciación entre empresarios y empleados, matizan la imagen romántica de las migraciones de los otavalos. Los duros inicios de la migración, un grado de movilidad muy alto y una fuerte concentración en el trabajo dejaban escaso margen para las prácticas religiosas.

"Habia unos meses que daba ganas de buscar (...) una iglesia (...) Los inviernos no trabajamos (...) pero llegaron unos momentos que ya no nos importaba si hay o no hay (...) No me interesaba ya (...) En veranos salíamos a las ferias y no habia tiempo ni para nada. Se trabajaba por la noche y de día descansábamos y luego por la tarde a trabajar (...) No he tenido tiempo para asistir y ya me dejé totalmente" (Magdalena)

Hay que enfatizar que cuando hablamos sobre la estabilización de los proyectos migratorios no nos referimos a prosperidad ni seguridad laboral completa. El trabajo de los otavalos está fuertemente condicionado por la necesidad de adaptación constante al mercado, tanto en cuanto a las mercancías ofrecidas como a las rutas comerciales que siguen los vendedores cada año. Sin embargo, con el tiempo, Villanueva se ha convertido en una base a la que se vuelve de los viajes comerciales. La mayoría de los otavalos ha conseguido además regularizar su situación administrativa.

Esta mayor estabilización coincide con el establecimiento de su propia entidad religiosa en la localidad. En el año 2009 crearon su congregación que, desde entonces, ha marcado profundamente la vida religiosa de la comunidad. La gran mayoría de los otavalos en Villanueva son evangélicos, aunque una parte de ellos se ha convertido ya en la emigración. Su iglesia forma parte de una entidad evangélica más amplia, a la que acuden personas de diversas nacionalidades, incluyendo población española. No obstante, los otavalos se reúnen por separado, denominando su congregación con el término Iglesia Kichwa. 
Las actividades religiosas en el seno de la entidad se ven profundamente marcadas por el nicho laboral compartido. El trabajo como comerciantes autónomos requiere, en el caso de la diáspora comercial de Otavalo, un enorme esfuerzo en la temporada de las ferias. En este periodo, de primavera a otoño, deben ganar lo suficiente para poder mantenerse en invierno, cuando las ganancias de las ventas en mercadillos locales tienen que ser complementadas con los ahorros. Ya que el periodo de las ferias es crítico para el sostenimiento de los individuos y las familias durante el resto del año, resulta fundamental que las prácticas religiosas no colisionen con el cumplimiento de los requisitos ocupacionales.

El caso de la diáspora comercial de Otavalo contrasta, por tanto, con múltiples investigaciones (Yang y Ebaugh, 2001; Casanova, 2007) que muestran que los migrantes, con frecuencia, asimilan las características de la vida religiosa de la sociedad receptora. Por ejemplo, en Estados Unidos las entidades de las minorías religiosas, tanto en el pasado entre los inmigrantes católicos y judíos como en la actualidad, suelen adoptar el modelo protestante congregacional.

En el caso de los kichwa otavalo la transformación de la vida religiosa se articula de manera distinta siguiendo, en parte, los requisitos de su peculiar inserción en el mercado laboral en el destino. En este contexto surge el fenómeno que denominamos "religiosidad segmentada", que hace referencia a la adherencia a nivel colectivo a significados y prácticas religiosas modificadas a partir de patrones de segregación étnica en el mercado de empleo. La sobrerrepresentación de migrantes de un origen específico en un nicho del mercado repercute en que su vida religiosa se vea transformada por los requisitos laborales.

Conviene aclarar que el concepto de la religiosidad segmentada permite analizar la influencia del mercado laboral en la religión, pero esto en ningún caso significa que la vida religiosa no sea afectada al mismo tiempo por otros factores. Como se evidenciará en el último apartado de resultados, el caso de los kichwa otavalo muestra, por ejemplo, una relevante continuidad con algunos de los patrones religiosos articuladas ya en la sociedad de origen. Por tanto para poder comprender el desarrollo de las prácticas y significados en la migración, debemos ubicar la influencia del mercado de empleo de destino en todo el contexto de movilidad. 


\section{ETNOESTRATIFICACIÓN DEL MERCADO LABORAL Y LA RELIGIOSIDAD SEGMENTADA}

El estudio de Tanya Korovkin (1998) defiende que la expansión evangélica en Otavalo contribuyó al declive de la agricultura familiar y a la expansión de la producción y comercio de textiles. La presente investigación muestra la relación inversa, aportando evidencias de cómo la actividad comercial también puede influir el ámbito religioso. A continuación veremos cómo la etnoestratificación presente en el mercado de trabajo en España interactúa con patrones religiosos. Para ello tenemos que analizar los principales requisitos laborales que se presentan en el destino.

El nicho laboral de los otavalos está fuertemente marcado por la estacionalidad. Cuando en abril se inicia la temporada de las ferias los otavalos empiezan un viaje que solo interrumpen, en el caso de los que se quedan en el sur de España, cuando vuelven los lunes o martes a la residencia habitual para descansar. Teniendo en cuenta las altas ventas de los fines de semana y el tiempo necesario para hacer los preparativos para el viaje y el propio traslado, queda muy poco tiempo para poder ocuparse de otras obligaciones no vinculadas con el trabajo. Durante los días de venta se duerme en el furgón, lo que repercute en que sea necesario poder volver para recuperar fuerzas. Hay localidades en las que las autoridades no ofrecen acceso de los vendedores a servicios básicos, lo que endurece las condiciones de trabajo aún más. Todo esto influye en que se haya consolidado un calendario laboral peculiar adaptado al calendario festivo de la sociedad española.

"Vengo un día, dos días para lavar la ropa (...) Trabajo por Jaén (...) tampoco puedo venir todos los días porque la gasolina cuesta y una vez que me voy, ya como empieza un jueves, termina la feria el domingo, entonces el lunes vengo a la casa (...) El miércoles ya empieza otra feria (...) y estamos saliendo de nuevo" (Magdalena)

Las ventas más altas tienen lugar de jueves a domingo, por lo que es imprescindible trabajar y, por tanto, estar de viaje durante estos días. Teniendo en cuenta que los fines de semana constituyen el periodo de trabajo más intenso, los informantes explican que tuvieron que reservar un tiempo entre semana para poder acudir a la entidad religiosa. A pesar de que el domingo es el día en el que se celebra el culto en el caso de la gran mayoría de las organizaciones cristianas de todo el mundo, en el caso de la población estudiada 
el cumplimiento de este patrón religioso se ve imposibilitado por sus obligaciones laborales. Para solucionar este problema, la Iglesia Kichwa ha trasladado su culto principal al martes. Se trata de la actividad principal realizada por la entidad cada semana. El cambio del día de la reunión más importante a lo largo de la semana permite a los kichwa otavalo cumplir con sus obligaciones religiosas en los pocos días de descanso que tienen entre ferias.

"Nosotros, por tema de trabajo, hemos escogido el día martes, no por escoger, sino que... porque Dios sabe que nosotros aqui estamos con este fuente de trabajo" (Franklin)

Hay que añadir que otras entidades religiosas en las que intentaron insertarse algunos de los componentes de la población analizada antes de fundar su propia congregación no ofrecían la posibilidad de realizar el culto otro día que no sea el domingo. En cambio, la iglesia más amplia en la que se inserta en la actualidad la Congregación Kichwa, aunque su reunión semanal tiene lugar los domingos, sí dio a los otavalos esta opción permitiéndoles reunirse aparte. La importancia atribuida al calendario religioso diferenciado queda plasmada en el hecho de que en el lenguaje cotidiano muchos otavalos denominen la organización más amplia en la que se insertan con el término de la "iglesia del domingo".

En los relatos recogidos, la creación de la Congregación Kichwa suele ser atribuida, de manera explícita, a la imposibilidad por parte de los otavalos de seguir el calendario de las prácticas religiosas existente en otras entidades evangélicas de la zona. Sin embargo, en muchos de los testimonios sobre la formación de la organización, se alude además a la afinidad cultural de sus componentes:

"En la parte de los kichwa somos un poco reservados (...) de que ves a otra persona de que no es como... tu misma... cultura (...) no te puedes soltar mucho, pero si estás dentro de tu misma cultura te sueltas y te ríes y sabes de lo que habla, te están entendiendo (...) A la misma vez nos enteramos de que unas hermanas decían sería muy bonito alabar en nuestro idioma (...) y a la misma vez también nos ayudaba que era el día martes, que el día martes nos podíamos congregar porque como la mayoría de los kichwa salimos a trabajar en las ferias entonces para venirnos un domingo, no podíamos (...) entonces el día martes nos iba muy bien (...) como el lunes por la noche regresamos y el martes estamos ya libres para poder ir a la iglesia" (Camila) 
Como ejemplifica la cita anterior, frecuentemente la argumentación laboral se entremezcla con alusiones a las diferencias lingüísticas y culturales. En las etapas iniciales del trabajo de campo se recolectaron varios testimonios que aludían a la posibilidad de hablar el kichwa como la razón clave de la fundación de su propia entidad. Es más, en la literatura especializada se describe frecuentemente la protección de la particularidad cultural y lingüística como uno de los fundamentos del éxito de las organizaciones evangélicas entre las poblaciones indígenas en el origen (Gros, 1999). La desestigmatización del kichwa fue, entre otros, facilitada por la traducción de la "Biblia" a este idioma en 1973.

Sin embargo, la observación participante llevada a cabo en las reuniones religiosas a lo largo de todo el periodo de investigación mostró una realidad que choca con la importancia atribuida a nivel discursivo al uso del kichwa. En primer lugar, las actividades religiosas se llevan a cabo en español. El kichwa constituye una parte mínima de estas, siendo utilizado tan solo para algunas alabanzas. En segundo lugar, la mayor parte de los otavalos de Villanueva no habla este idioma, aunque sí lo entiende. Pese a que la diferenciación lingüística parece tener un valor identitario, el castellano es claramente el idioma ampliamente predominante en las actividades religiosas.

Habría que añadir que además existen relatos que se desvinculan de argumentos étnicos enfatizando que la única razón para la creación de la entidad es el hecho de compartir el mismo trabajo. En estos discursos se defiende que, si tuviesen la opción de asistir a las reuniones del domingo, no habría separación en términos organizacionales.

"No es sólo para los kichwa (...) Si quiere puede asistir cualquier persona. Están las puertas abiertas. Es solamente por el trabajo" (Fabián)

La necesidad de adaptación de las prácticas religiosas a los requisitos laborales se plasma no solo en el calendario semanal sino también en las festividades anuales más destacadas. Algunas de las celebraciones más relevantes para la mayoría de las ramas del cristianismo desaparecen del calendario festivo de la Iglesia Kichwa. En su lugar surgen otras celebraciones que se vinculan estrechamente con el perfil laboral adoptado en el destino. 
Destaca el hecho de que los otavalos no puedan celebrar la Semana Santa. A pesar de que se trata de un periodo especialmente importante en el calendario de las festividades anuales, en el caso de los kichwa otavalo, su celebración se ve imposibilitada por los requisitos de su trabajo. La Semana Santa tiene lugar una vez se ha iniciado la temporada de trabajo. Además, se trata de un periodo caracterizado por ventas elevadas por lo que para los informantes es un tiempo de esfuerzo intenso ya que no pueden permitirse prescindir de los ingresos que genera.

"Aquí no hacemos nada (...) Desde que hemos llegado aquí a España, la Semana Santa la pasamos trabajando. Entonces no lo hemos hecho nosotros, nadie de nuestra cultura (...) Hemos intentado reunirnos entre todos y hacerlo pero casi siempre el trabajo que no nos ha permitido hacerlo y nosotros y mi familia no lo hemos hecho nunca desde que estamos acá en España" (Diana)

La adopción de la perspectiva de la religión "vivida" hizo posible la apreciación de transformaciones de la vida religiosa como las mencionadas anteriormente. Si nos limitásemos a comprobar el cumplimiento de los ritos preestablecidos por las instituciones dominantes, es bastante probable que pudiéramos perder de vista este tipo de prácticas. Para realizar una encuesta sobre la religiosidad de los migrantes en España partiendo de las definiciones más extendidas de las prácticas organizacionales de las principales corrientes del cristianismo deberíamos incluir preguntas referentes a la participación en el culto dominical y la celebración de fiestas como la Semana Santa. Si nos atuviéramos a indicadores como los mencionados, los evangélicos kichwa otavalo podrían parecer poco religiosos, al menos en lo referente a su participación en las religiones organizadas.

El uso de indicadores rígidos de la religiosidad podría llevar además a la invisibilización de nuevas prácticas religiosas. Partiendo desde la perspectiva de la religión "vivida", pudimos captar el surgimiento de la celebración del principio y final de la temporada de las ferias. Ambas se realizan de manera anual. La primera, como preparación para el viaje, sirve para que todos se reúnan en un culto especial en el que se pide protección para toda la temporada de trabajo. Normalmente, después del culto, se da paso a una parte menos formal. Las participantes preparan comida en casa y esta se consume en el espacio que brinda la Iglesia. Se invita también a los, 
pocos, otavalos católicos de la localidad a asistir a esta celebración, adaptada, en definitiva, a una forma de trabajo compartida a nivel colectivo. Los informantes explican la realización de este culto aludiendo a los riesgos asociados a la constante movilidad durante la temporada de trabajo. De igual manera, en otoño, de nuevo todos se reúnen organizando un culto que denominan "Acción de Gracias", en el que agradecen la posibilidad de regresar a sus hogares después del periodo de esfuerzo intensivo.

"El invierno nos juntamos y [en] abril, mayo, todos casi desaparecen de aquí (...) y empieza el viaje (...) todos viajando. Entonces con esta idea nosotros nos hemos puesto a hablar (...) los hermanos kichwa: "¿Por qué no hacemos un culto a encomendarnos al Señor por nuestra salida?» (...) que nos cuide durante toda nuestra temporada (...) Y ha salido... creo que faltaron 4, 5 personas que no podian venir, y de alli fueron todos, todos, todos, pero todos los que vivimos aquí los cristianos y los no cristianos (...) A lo mejor nuestro trabajo es un poco riesgoso, porque tú estás en la feria de toda la noche, recoges a las 5-6 de la madrugada y tienes que hacer un viaje nuevamente a donde sea, estás viajando con el sueño, estás viajando y siempre viajando (...) entonces nuestro viaje en la carretera corres siempre riesgo (...) Entonces esto hemos hecho al salir (...) ahora este martes queremos hacer el regreso. ¿Por qué? Porque hemos venido, gracias a Dios, tranquilos" (David)

La observación participante permitió constatar que se trata de celebraciones que, a nivel general, conservan el formato presente en las celebraciones semanales. Como es habitual se inicia con la alabanza, para luego pasar a la predicación. Lo que es diferente es la segunda parte de estas reuniones, en la que en el espacio de la iglesia se realiza una comida compartida. En estas ocasiones el uso del kichwa está más presente. Pese a la falta de la capacidad de hablar en este idioma de la mayoría de los miembros de la iglesia, se suele realizar una oración dirigida por parte de los pocos componentes que tienen la capacidad de expresarse en kichwa. Se trata de ocasiones especiales que permiten la reafirmación de los elementos de la cultura de origen. Esta cuestión conecta con la compleja relación entre la religión y la identidad, que abordaremos en el siguiente apartado. 


\section{LA RELIGIÓN Y LOS PROCESOS DE NEGOCIACIÓN IDENTITARIA}

La fundación de una entidad religiosa propia, por una parte, lleva a la reafirmación de la auto-identificación como otavalos. No obstante, por otra, genera una división dentro de la comunidad en Villanueva ya que una pequeña minoría de los otavalos asentados en la localidad no es evangélica. En el presente artículo, centrado en el tema de la religiosidad segmentada, señalamos tan solo algunos de los elementos clave de la ambigua relación que se articula entre la religión y la etnicidad. Se defiende que la inserción laboral es importante para comprender los patrones religiosos en el país de destino, pero el análisis de su influencia no debe perder de vista otros factores que repercuten en cómo se vive la religión.

El estudio capta cómo las prácticas religiosas pueden contribuir al fomento de la identidad étnica en el contexto migratorio. El nivel de emoción observado durante las reuniones de los martes, pero sobre todo durante las celebraciones más importantes a lo largo del año, apoyan la tesis de la capacidad del hecho religioso de provocar "efervescencia colectiva". Este término, acuñado por Durkheim (2001), permite explicar cómo los ritos religiosos pueden generar sentimientos muy fuertes de pertenencia a la comunidad, fomentando así la cohesión social. Durkheim argumentaba que los rituales, tanto religiosos como de otra naturaleza, cumplen con este propósito si siguen los siguientes requisitos:

(1) El encuentro en un mismo lugar.

(2) La existencia de límites físicos que marcan a la vez la diferenciación del mundo externo.

(3) La realización de los mismos actos.

(4) La experimentación de sentimientos compartidos.

A raíz de la "efervescencia colectiva" las creencias compartidas y el sentimiento de pertenencia a una misma comunidad se ven reafirmadas. Casi todas las mujeres kichwa otavalo acuden a la iglesia con la vestimenta femenina típica de Otavalo, introduciendo así elementos de la cultura material que contribuyen a que los ritos tengan un componente identitario muy potente. La importancia de la cohesión grupal de la entidad formada por los otavalos puede ser ilustrada a través de la siguiente cita. 
"Todos los componentes de la Iglesia Kichwa somos un miembro" (Franklin)

Hay que enfatizar que la expansión de las iglesias evangélicas en el origen se fundamentó en gran medida en el reconocimiento de la importancia de la identificación étnica (Gros, 1999). En este sentido, pese a la modificación de algunas pautas religiosas a raíz de la actividad laboral, existe una importante continuidad con las prácticas religiosas del origen. Una vez consolidadas las estructuras organizativas separadas a través del establecimiento de la Iglesia Kichwa, surgen nuevas pautas de comportamiento compartido que siguen el principio étnico. Entre otras actividades, dentro de la iglesia se realizan reuniones de bordado que reavivan la tradición artesanal de Otavalo produciendo manualmente el bordado típico de las blusas femeninas del origen. Se mezclan así las prácticas religiosas, como la lectura grupal de los pasajes bíblicos y la oración, con otras que se alejan de los ritos de fe. Se pretende compartir conocimientos necesarios para la elaboración del ropaje típico, influyendo en la perduración de las actividades artesanales de Otavalo.

De forma periódica se organiza, además, la práctica denominada el "retiro kichwa". Se trata de una salida grupal de varios días a otra localidad dedicada a la oración y la reflexión. A pesar de que dentro de la entidad evangélica más amplia en la que se inserta la Iglesia Kichwa se realizan retiros para hombres y para mujeres en los que participan evangélicos de múltiples nacionalidades, es el "retiro kichwa" el que presenta mayor inscripción de los otavalos. El hecho de que exista un retiro especial para los kichwa atestigua de nuevo el surgimiento de prácticas religiosas y de su ajuste a las necesidades específicas de esta población. Los migrantes kichwa otavalo organizan también en ocasiones salidas sin mediación de la iglesia, reuniéndose en descampados en las cercanías de Villanueva.

Aparte del "retiro kichwa", en el periodo de invierno se intensifica la dedicación a las prácticas religiosas. Fuera de la temporada alta de trabajo es mucho más fácil participar en diversas iniciativas realizadas a nivel colectivo. Los otavalos pueden incorporarse en mayor medida a las actividades realizadas por la entidad evangélica en la que se inserta su iglesia, asistiendo, por ejemplo, al culto dominical. No obstante, se organizan otras actividades dedicadas específicamente a los otavalos. Por ejemplo, a lo largo de esta investigación, empezaron a realizarse reuniones semanales de los hombres otavalos. 
A pesar de las pautas religiosas descritas más arriba, estaríamos equivocados si asumiéramos que la religión, de manera unidireccional, reafirma la identificación étnica. La investigación ha captado las divisiones dentro de la comunidad que ha generado la fundación de la iglesia.

Las actividades dentro de la Iglesia Kichwa eran dominadas inicialmente por los otavalos que eran evangélicos ya en el origen. Con el tiempo, el grupo empezó a expandirse a raíz de conversiones estimuladas por la intensa actividad de los miembros de la iglesia. La prohibición del baile y del consumo de alcohol adquirió importancia como marcador identitario de los evangélicos, llevando a tensiones en torno a las formas de celebrar las reuniones de ocio que antes acogían a todos los otavalos de la localidad independientemente de su religión. Observamos una creciente diferenciación entre los evangélicos y los otavalos que, siguiendo la terminología utilizada en la iglesia, "viven en el mundo".

"Cuando tenemos nuestras celebraciones (...) de Navidad, de Año Nuevo o... un cumpleaños, lo hacemos (...) solamente nosotros y no es porque no queremos invitarles a esta... a otra gente, nuestra gente ino? (...) sino que (...) tienen otra manera (...) de ver las cosas (...) Nos gustaría muchas veces invitarles a ellos pero (...) ellos creen ser diferentes, ¿no? Que ellos están en un camino muy diferente y que nosotros somos los del mundo" (Fernando, católico)

La memoria histórica, el nicho laboral compartido y las prácticas religiosas unen a los participantes de la Congregación Kichwa, pero la creación de la iglesia abrió nuevos espacios de negociación de la identidad, contribuyendo a fronteras sociales alternativas a las étnicas. Surge una división entre los otavalos católicos y evangélicos que antes del establecimiento de la entidad no era tan patente ya que la precariedad laboral predominante se traducía en la concentración en el trabajo dejando poco espacio para las prácticas religiosas. A las divisiones emergentes se une la mediación de la entidad en el establecimiento de vínculos con co-creyentes españoles y de otras nacionalidades, fomentando el sentimiento de pertenencia a una comunidad en la que las identificaciones étnicas pierden peso a favor de las categorías identitarias religiosas.

La religión, por un lado, reafirma la etnicidad pero, por otro, aumenta la diversificación de las redes sociales de los otavalos, a la 
vez que introduce una división interna al abogar por el abandono de todo lo que tiene que ver con "el mundo". En el transcurso de la investigación, a los otavalos que eran evangélicos en Ecuador se unieron otros convertidos ya en la emigración. Hoy en día la población kichwa otavalo de Villanueva está formada en su gran mayoría por evangélicos. De esta manera el éxito evangélico en el origen llega a plasmarse también en la diáspora. En Ecuador la expansión de las entidades evangélicas se suele vincular, entre otros, a la exitosa incorporación de las pautas culturales asociadas a la población indígena, la actividad asistencial desempeñada por muchas organizaciones y a la crisis de las pautas religiosas tradicionales (Korovkin, 1998; Gros, 1999). Todos estos patrones se pueden observar también en la diáspora. El caso analizado muestra que en el contexto migratorio las organizaciones evangélicas pueden, además, llegar a caracterizarse por un elevado grado de plasticidad que les permite adaptarse a los requisitos de trabajo de los otavalos en la emigración. Sin duda no se trata del único factor que explica los elevados niveles de adherencia religiosa y las conversiones. No obstante, los patrones de la religiosidad segmentada contribuyen a comprender el surgimiento de la Iglesia Kichwa en Villanueva, así como la articulación de las prácticas religiosas de sus componentes.

\section{CONCLUSIONES}

A pesar de que diversos estudios (Yang et al., 2001; Casanova, 2007) muestran la asimilación de los patrones de religiosidad de la sociedad de acogida por parte de los migrantes, incluyendo a los pertenecientes a las minorías religiosas, el análisis desde la perspectiva de la religión "vivida" del caso de los kichwa otavalo atestigua que la transformación de la vida religiosa en el destino puede seguir una vía diferente. En lugar de adoptar las pautas religiosas predominantes, sus prácticas se ven parcialmente modificadas por los requisitos del nicho laboral ocupado en el contexto migratorio.

El elevado grado de homogeneidad laboral lleva al desarrollo de lo que denominamos "religiosidad segmentada". El concepto hace referencia a la adherencia a nivel colectivo a significados y prácticas religiosas transformadas a raíz de patrones de segregación en el mercado laboral. El término permite captar la influencia de la 
sobrerrepresentación de migrantes de un origen en un nicho laboral específico en su vida religiosa.

Para comprender la forma peculiar de la inserción de los kichwa otavalo en el mercado laboral en España hay que tener en cuenta el contexto de origen y, específicamente, el desarrollo de la economía étnica en Otavalo. Las migraciones generadas por la demanda global de lo "auténticamente indígena" siguen hoy estando marcadas por la especialización en el comercio. Los kichwa otavalo de Villanueva, pese a haber abandonado el empresariado transnacional, muestran aún un muy alto nivel de concentración laboral en el comercio en las ferias. De este modo siguen ocupando un lugar específico en el mercado de empleo etnoestratificado existente en diversos países de inmigración (Midtbøen, 2014; Koopmans, 2016).

El desarrollo de la religiosidad segmentada se plasma en el ajuste de las prácticas religiosas a los requisitos de trabajo en las ferias. La estacionalidad de su ocupación implica que deben concentrar sus esfuerzos laborales en el periodo de primavera a otoño, trabajando de manera más intensa durante los fines de semana. Su calendario laboral repercute en que el culto del domingo, el día de la semana más celebrado por la mayoría de los cristianos en el mundo, no pueda ser incorporado en las prácticas religiosas de la población estudiada. Como consecuencia, no solo se traslada el culto principal al martes sino que, además, se introducen modificaciones profundas en las celebraciones anuales más importantes. Las fiestas que, como la Semana Santa, tienen lugar una vez iniciada la temporada de trabajo, dejan de ser celebradas. Paralelamente surgen otras, entre las cuales hay que destacar el culto del inicio y del final del periodo de trabajo en las ferias. Este peculiar calendario religioso gira en torno a las pautas sociales marcadas por el calendario festivo de la sociedad receptora. A raíz del establecimiento de la Iglesia Kichwa y el surgimiento de estructuras organizativas separadas que se adaptan al nicho laboral ocupado, se generan nuevas prácticas religiosas, como el "retiro kichwa", que fomentan adicionalmente la cohesión grupal.

Los resultados de la investigación aportan evidencias sólidas que permiten cuestionar las visiones estáticas de la religión que, en discursos anti-inmigración en Europa, son frecuentemente utilizadas para argumentar la existencia de barreras insuperables, arraigadas en diferencias culturales naturalizadas, entre migrantes y sociedades de acogida. Como muestra el presente estudio, las prácticas y 
significados religiosos pueden cambiar en un periodo de tiempo muy corto, incluso a raíz de condicionantes aparentemente tan alejados del ámbito religioso como el mercado laboral, poniendo en duda de esta manera las conceptualizaciones esencialistas de la religión.

\section{REFERENCIAS BIBLIOGRÁFICAS}

Ammerman, N. T. (2016). Lived Religion as an Emerging Field: An Assessment of Its Contours and Frontiers. Recuperado de https://dcommon. bu.edu/handle/2144/19161.

Atienza de Frutos, D. (2009). Viaje e identidad. La génesis de la élite kichwa-otavaleña en Madrid, España. Quito: Abya-Yala.

Bollard, A., McKenzie, D., Morten, M., y Rapoport, H. (2011). Remittances and the Brain Drain Revisited: The Microdata show that more Educated Migrants remit more. The World Bank Economic Review. doi:10.1093/ wber/lhr013.

Caicedo, L. P. (2010). Los Kichwa-Otavalos en Bogotá. En A. Torres (Ed.), Niñez Indígena en Migración: Derechos en Riesgo y Traumas Culturales (pp. 139-226). Quito: FLACSO, Sede Ecuador.

Carrasco, C., y García, C. (2015). Efectos de la Crisis en la Estructura Ocupacional y la Biografia Laboral de la Población Inmigrante. Migraciones. Publicación del Instituto Universitario de Estudios Sobre Migraciones, 37, 75-96.

Casanova, J. (2007). La Inmigración y el Nuevo Pluralismo Religioso: una Comparación Unión Europea/Estados Unidos. Revista CIDOB D'afers Internacionals, 77, 13-39.

Cebrián, J. A., Jiménez, B. C., y Resino R. M. (2016). Inmigrantes Emprendedores Durante la Crisis Económica. Estudio de Casos en la Ciudad de Madrid. Migraciones. Publicación del Instituto Universitario de Estudios sobre Migraciones, 40, 63-92.

Célleri, D., y Jüssen, L. (2012). Solidaridad Étnica y Capital Social. El Caso de los Comerciantes Migrantes Kichwa-Otavalo en Madrid y La Compañía. Procesos: Revista Ecuatoriana de Historia, 36, 143-168.

Colic-Peisker, V. (2010). Free Floating in the Cosmopolis? Exploring the Identity-Belonging of Transnational Knowledge Workers. Global Networks, 10(4), 467-88. doi:10.1111/j.1471-0374.2010.00298.x.

Colloredo-Mansfeld, R. (1998). 'Dirty Indians', Radical Indígenas and the Political Economy of Social Difference in Modern Ecuador. Bulletin of Latin American Research, 17(2), 185-205.

Conejo, M., Yamberla, J., y Cachiguango, I. (2003). Los quichua-otavalo: economía e identidad. En T. Carrasco, D. Iturralde y J. Uquillas (Ed.), Doce experiencias de desarrollo indígena en América Latina (pp. 193213). Quito: Abya-Yala. 
Durkheim, É. (2001). The Elementary Forms of Religious Life. Oxford: Oxford University Press.

Foner, N., y Alba, R. (2008). Immigrant Religion in the U.S. and Western Europe: Bridge or Barrier to Inclusion? International Migration Review, 42(2), 360-392.

Gros, C. (1999). Evangelical Protestantism and Indigenous Populations. Bulletin of Latin American Research, 18(2), 175-197.

Gumucio, C. P. (2002). Religion and the Awakening of Indigenous People in Latin America. Social Compass, 49(1), 67-81.

Hall, D. (1997). Lived Religion in America: Towards a History of Practice. Princeton: Princeton University Press.

Heath, A. F., Rothon, C., y Kilpi, E. (2008). The Second Generation in Western Europe: Education, Unemployment, and Occupational Attainment. Annu. Rev. Sociol, 34, 211-235.

Huarcaya, S. M. (2011). Othering National Identity Alterity and Indigenous Activism in Otavalo, Ecuador. Michigan: University of Michigan.

Johnston, R., Khattab, N., y Manley, D. (2015). East versus West? OverQualification and Earnings among the UK's European Migrants. Journal of Ethnic and Migration Studies, 41(2), 196-218. doi:10.1080/136918 3X.2014.935308.

Koopmans, R. (2016). Does Assimilation Work? Sociocultural Determinants of Labour Market Participation of European Muslims. Journal of Ethnic and Migration Studies, 42(2), 197-216. doi:10.1080/136918 3X.2015.1082903.

Korovkin, T. (1998). Commodity Production and Ethnic Culture: Otavalo, Northern Ecuador. Economic Development and Cultural Change, 47(1), 125-154.

Kyle, D. (2000). Transnational Peasants: Migrations, Networks, and Ethnicity in Andean Ecuador. Baltimore and London: Johns Hopkins University Press.

Kyle, D. (2001). La diáspora del comercio otavaleño: Capital social y empresa transnacional. Quito: CAAP. http://flacsoandes.org/dspace/hand le/10469/4892

Lalander, R. 2010. Between Interculturalism and Ethnocentrism: Local Government and the Indigenous Movement in Otavalo-Ecuador. Bulletin of Latin American Research, 29(4), 505-521.

McGuire, M. (2008). Lived Religion: Faith and Practice in Everyday Life. Oxford: Oxford University Press.

Meisch, L. (2002). Andean Entrepreneurs: Otavalo Merchants and Musicians in the Global Arena. Austin: University of Texas Press.

Midtbøen, A. H. (2014). Discrimination of the Second Generation: Evidence from a Field Experiment in Norway. Journal of International Migration and Integration, December, 1-20.

Ordóñez, A. (2008). Migración Transnacional de los Kichwa Otavalo y la Fiesta del Pawkar Raymi. En A. Torres y J. Carrasco (Ed.), Al Filo de La 
Identidad: La Migración Indígena En América Latina (pp. 69-88). Quito: FLACSO.

Portes, A., Guarnizo, L. E., y Haller, W. J. (2002). Transnational Entrepreneurs: an Alternative Form of Immigrant Economic Adaptation. American Sociological Review, 67(2), 278-98.

Ruiz, A. (2015). Tejedores de mapas. Una familia kichwa otavaleña en la migración transoceánica. Quito: FLACSO.

Schielke, S., y Debevec, L. (2012). Ordinary Lives and Grand Schemes: an Anthropology of Everyday Religion, Vol. 18. New York, Oxford: Berghahn Books.

Solé, C., Parella, S., y Cavalcanti, L. (2007). El Empresariado Inmigrante En España. Barcelona: Fundación La Caixa. Recuperado de

http://www.fundacion.lacaixa.es/StaticFiles/StaticFiles/39d4ffd999ea2110V gnVCM200000128cf10aRCRD/es/es21_inx_esp.pdf.

Torres, A. (2004). El Espejismo de la Igualación: Comunidad, Clase y Etnia en la Emigración de los Kichwa Otavalo. Recuperado de

http://www.flacso.org.ec/docs/at_espejismo.pdf.

Woodhead, L. (2014). Strategic and Tactical Religion. En L. Woodhead, N. M. Dessing, J. S. Nielsen y N. Jeldtoft (Ed.), Everyday Lived Islam in Europe (pp. 143-154). Aldershot: Ashgate Publishing, Ltd.

Woodhead, L., Dessing, N. M, Nielsen, J. S., y Jeldtoft, N. (2014). Everyday Lived Islam in Europe. Ashgate Publishing, Ltd.

Yang, F. y Ebaugh, H. (2001). Transformations in New Immigrant Religions and Their Global Implications. American Sociological Review, 66, 269288.

Zhou, M. (2004). Revisiting Ethnic Entrepreneurship: Convergencies, Controversies, and Conceptual Advancements. International Migration Review, 38(3), 1040-1074.

Zolberg, A. R., y Woon, L. L. (1999). Why Islam Is like Spanish: Cultural Incorporation in Europe and the United States. Politics \& Society, 27(1), $5-38$. 